\title{
THE RIESZ BASIS PROPERTY OF THE SYSTEM OF ROOT VECTORS FOR THE EQUATION OF A NONHOMOGENEOUS DAMPED STRING: TRANSFORMATION OPERATORS METHOD*
}

\author{
MARIANNA A. SHUBOV ${ }^{\dagger}$
}

\begin{abstract}
We prove the spectral decomposition theorem for a class of nonselfadjoint operators in a Hilbert space. These operators are the dynamics generators for the systems governed by 1-dimensional hyperbolic equations with spacially nonhomogeneous coefficients containing first order damping terms and subject to linear nonselfadjoint boundary conditions. These equations and boundary conditions describe, in particular, a spacially nonhomogeneous string subject to a distributed viscous damping and damped at the boundary points. Our main result leading to the spectral decomposition is the fact that the root vectors (eigenvectors and associated vectors together) of the above operators form Riesz bases in the corresponding energy spaces. Our proofs are based on the transformation operator method suggested in our previous work [1]. In that work, we extended the classical concept of transformation operators to the equation of a damped string. Originally, this concept was developed by I.M. Gelfand, B.M. Levitan and V.A. Marchenko for 1-dimensional Schrodinger equation in connection with the inverse scattering problem. Using the transformation operator method, we reduce the problem of Riesz basis property for the root vectors to the similar problem for a system of nonharmonic exponentials whose complex frequencies are precisely the eigenvalues of our operators. The latter problem is solved based on the spectral asymptotics and known facts about the families of nonharmonic exponentials.
\end{abstract}

1. Introduction. In the present paper, we develop the spectral analysis for a class of nonselfadjoint operators and related nonselfadjoint quadratic operator pencils. These operators are the dynamics generators for a spacially nonhomogeneous damped string with a distributed viscous damping which is subject to a class of nonselfadjoint boundary conditions containing two complex parameters. Depending upon the values of the parameters, these conditions describe either loss or gain of energy through the boundary. Our main result is the fact that the aforementioned operators are Riesz spectral. The result, crucial for the proof, is the Riesz basis property of the root vectors of these operators in the corresponding energy spaces. To obtain this result, we apply the transformation operator method suggested in our work [1]. Particular cases of this spectral problem were studied in [4-10] (see a more detailed discussion in Remark 2.1 below). For the general case of the string containing dampings both in the equation and in the boundary conditions, the Riesz spectral property result has not been established before. We expect that the method suggested in this paper is quite general and can be applied to a wide class of systems governed by hyperbolic equations including various beam models.

We point out that there exists an extensive literature on the geometric properties of root vectors of various special classes of nonselfadjoint operators (e.g., see[11-21]). In particular, we mention deep results by A.S.Marcus [16-19],V.I.Matsaev [18, 19], and M.S.Agranovich [11, 12]. However, the results that we obtained by the transformation operator method and which we present in this paper cannot be derived from the aforementioned general results.

The paper is organized as follows. In Section 2, we describe the problem, recall the notion of a Riesz spectral operator, and state our main result - Theorem 2.1. In Section 3 , we formulate the necessary results from our work [1] devoted to the transformation operator method (Theorem 3.3). To this end, we recall the spectral asymptotics and

\footnotetext{
* Received July 7, 1998; revised April 10, 1999.

${ }^{\dagger}$ Mathematical Department, Texas Tech University, Lubbock, TX 79409, USA (mshubov@math. ttu.edu).
} 
asymptotic representations for the special solutions of the string equation from our papers $[4,26]$. Sections 4-6 are devoted to the proof of our main result. This proof splits into several steps. In each step, we obtain intermediate results which might be of interest in themselves. In Section 4, we use the transformation operator method to establish the Riesz basis property of the set of special solutions of the corresponding pencil equation. In Section 5, we define the generalized Jost solutions evaluated at the spectral points. Finally, in Section 6, we complete the proof of the Riesz basis property of the root vectors. In this proof, we employ some results presented in our earlier papers [2] and [5], and also use the classical theorems of M.V. Keldysh and N.K. Bari [28].

2. Statement of Problem. Let us consider the equation of a nonhomogeneous damped string

$$
w_{t t}(x, t)+2 \mathbf{d}(x) w_{t}(x, t)+L w(x, t)=0,
$$

where $L$ is the Sturm-Louisville operator defined on any smooth function $\varphi$ by the formula:

$$
(L \varphi)(x)=-\frac{1}{\rho(x)} \frac{d}{d x}\left(p(x) \frac{d}{d x} \varphi(x)\right)+q(x) \varphi(x) .
$$

We assume that $w(x, t)$ is defined for $x \in[0, a], 0<a<\infty, t \geq 0$. The coefficients $\mathbf{d}$ ("viscous damping"), $\rho$ (density of the string), $p$ (modulus elasticity) and $q$ (rigidity of external harmonic force) are subject to the following conditions:

$$
\rho, p \in W_{1}^{2}(0, a), \mathbf{d} \in W_{1}^{1}(0, a), q \in L^{\infty}(0, a) ; \rho(x), p(x) \geq C>0 \text { for } x \in[0, a] ;
$$

(We use the standard notation $W_{m}^{l}$ for the Sobolev space of functions having weak derivatives up to the order $\ell$ in the space $L^{m}$.)

REMARK 2.1. We do not impose on $\mathbf{d}$ the condition: $\mathbf{d} \geq 0$ since all our results are valid without this assumption. However, we call this coefficient "viscous damping." Certainly,this phrase is some abuse of terminology because, rigorously speaking, only a nonnegative coefficient $\mathbf{d}$ can be called viscous damping.

We impose the following boundary conditions:

$$
\left(w_{x}+k w_{t}\right)(0, t)=\left(w_{x}+h w_{t}\right)(a, t)=0, \quad h, k \in \mathbb{C} \cup\{\infty\} .
$$

Depending upon the values of the parameters $h$ and $k$, conditions (2.4) describe different physical phenomena which are mentioned below. The case $h=k=\infty$ (the Dirichlet boundary conditions) corresponds to the vibrations of the string clamped at both ends. The case $h=k=0$ (the Neumann boundary conditions) corresponds to the vibrations of the string with free ends. If $k=\infty$ and $h=1$, then we have the so-called mixed boundary condition at the right end. (If the coefficients of Eq.(2.1) are extended from $[0, a]$ to $[0, \infty]$ by the rule: $p(x)=p(a), \rho(x)=\rho(a)$ for $x \geq a$ and $\mathbf{d}(x)=q(x)=0$ for $x \geq a$ and if the problem is considered on the entire semiaxis $[0, \infty]$, then this boundary condition is equivalent to the Sommerfeld radiation condition.) This condition is used for the description of the resonance phenomenon in the scattering of elastic waves on the semi-infinite string. The boundary conditions with $h=-k=1$ are used in the description of the resonance scattering of acoustical waves on the entire axis by an obstacle concentrated on the interval $[0, a]$. 
As is well known, Eq. (2.1) can be represented in the form of the first order evolution equation for a 2-component function:

$$
W_{t}(x, t)=i(\mathfrak{L} W)(x, t), \quad W(x, t)=\left(\begin{array}{c}
w(x, t) \\
w_{t}(x, t)
\end{array}\right)
$$

where $\mathfrak{L}$ is the matrix differential expression

$$
\mathfrak{L}=-i\left(\begin{array}{cc}
0 & 1 \\
L & -2 \mathfrak{d}
\end{array}\right)
$$

with $L$ being defined in (2.2). Eq. (2.5) with boundary conditions (2.4) defines a strongly continuous semigroup of transformations in the Hilbert space of 2-component initial data. This Hilbert space (the energy space) $\mathcal{H}$ is a closure of smooth, 2component functions in the standard energy norm:

$$
\|W\|_{\mathcal{H}}^{2}=1 / 2 \int_{0}^{a}\left[p(x)\left|w_{0}^{\prime}(x)\right|^{2}+q(x) \rho(x)\left|w_{0}(x)\right|^{2}+\rho(x)\left|w_{1}(x)\right|^{2}\right] d x .
$$

Note that for $k=\infty$, the energy space $\mathcal{H}$ is defined as a closure of smooth functions satisfying an additional condition: $w_{0}(0)=0$. Let us define the operator $\mathfrak{L}_{h k}$ as a matrix differential operator given by expression $(2.6)\left(\mathfrak{L}_{h k}=\mathfrak{L}\right)$ on the domain:

$$
D\left(\mathfrak{L}_{h k}\right)=\left\{W=\left(w_{0}, w_{1}\right) \in \mathcal{H}: \mathfrak{L} W \in \mathcal{H} ;\left(w_{0}^{\prime}+k w_{1}\right)(0)=0,\left(w_{0}^{\prime}+h w_{1}\right)(a)=0\right\} .
$$

Since $\rho(x)$ and $p(x)$ are bounded functions that stay away from zero, the condition $\mathfrak{L} W \in \mathcal{H}$ simply means that $w_{0} \in W_{2}^{2}(0, a)$ and $w_{1} \in W_{2}^{1}(0, a)$.

REMARK 2.2. The operator $\mathfrak{L}_{h k}$ is the generator of the aforementioned, strongly continuous semigroup. This fact can be obtained as an immediate corollary of our results stated below in Theorem 3.1, Theorem 6.2, and by well known results of the semigroup theory

It is the operator $\mathfrak{L}_{h k}$ which is our main object of interest. Using the approach developed in [1], we prove our results not only for the operator $\mathfrak{L}_{h k}$ but also for the adjoint operator $\mathfrak{L}_{h k}^{*}$. It is convenient to define it now. $\mathfrak{L}_{h k}^{*}$ is defined by the differential expression

$$
\mathfrak{L}_{h k}^{*}=-i\left(\begin{array}{cc}
0 & 1 \\
-L & 2 \mathfrak{d}
\end{array}\right)
$$

on the domain:

$$
D\left(\mathfrak{L}_{h k}^{*}\right)=\left\{W \in \mathcal{H}: \mathfrak{L}_{h k}^{*} W \in \mathcal{H}, \quad\left(w_{0}^{\prime}-\bar{k} w_{1}\right)(0)=0, \quad\left(w_{0}^{\prime}-\bar{h} w_{1}\right)(a)=0\right\} .
$$

Note that both operators are nonselfadjoint, simple, maximal (dissipative for $R e h \geq$ $0, R e k \leq 0$, and $\mathfrak{d} \geq 0$ ) operators in $\mathcal{H}$ (see [2]). (We recall that a nonselfadjoint operator $A$ is simple if $A$ and $A^{*}$ do not have a common invariant subspace on which they coincide.) The resolvent of each operator is compact, and therefore the spectrum is discrete. 
We note that the operator $\mathfrak{L}_{h k}$ is nonselfadjoint for two reasons: first, due to the difference in the signs before the damping terms $2 \mathfrak{d}$ (see (2.6), (2.9)) and, secondly, due to the difference in the boundary conditions if at least one of the parameters, $h$ or $k$, is not purely imaginary.

As was previously mentioned, our goal is to obtain the spectral decomposition theorem for the operators $\mathfrak{L}_{h k}$ and $\mathfrak{L}_{h k}^{*}$. It is well known that at the present moment there is no general spectral theory for nonselfadjoint operators in a Hilbert space. Such a theory has been developed only for some special classes of nonselfadjoint operators, and one of these classes is the class of spectral operators [3]. Below we recall the necessary definitions in the form convenient for the present exposition.

Let $H$ be a complex Hilbert space and $\mathcal{R}(H)$ be the class of bounded linear operators in $H$.

Definition 2.1. a) A sequence of vectors $\left\{\psi_{n}\right\}_{n \in \mathbb{Z}} \subset H$ is called a Riesz basis if there exists an operator $A \in \mathcal{R}(H)$ such that $A^{-1} \in \mathcal{R}(H)$ and the system $\left\{A \psi_{n}\right\}_{n \in \mathbb{Z}}$ forms an orthonormal basis in $H$. A is called an orthogonalizer.

b) For any Riesz basis, there exists a unique biorthogonal Riesz basis $\left\{\psi_{n}^{*}\right\}_{n \in \mathbb{Z}}\left(\left(\psi_{n}, \psi_{m}^{*}\right)_{H}=\delta_{n m}\right)$ with the orthogonalizer $\left(A^{-1}\right)^{*}$.

DEFINITION 2.2. Let $\left\{\psi_{n}\right\}_{n \in \mathbb{Z}}$ be a Riesz basis in $H$ and $\left\{\psi_{n}^{*}\right\}_{n \in \mathbb{Z}}$ be the biorthogonal basis; let $\left\{\lambda_{n}\right\}_{n \in \mathbb{Z}}$ be a sequence of complex numbers. A linear operator $S$ in $H$ defined by the formula

$$
S \varphi=\sum_{n \in \mathbb{Z}} \lambda_{n}\left(\varphi, \psi_{n}^{*}\right)_{H} \psi_{n}
$$

on the dense domain

$$
D(S)=\left\{\varphi \in H: \sum_{n \in \mathbb{Z}}\left|\lambda_{n}\right|^{2}\left|\left(\varphi, \psi_{n}^{*}\right)_{H}\right|^{2}<\infty\right\}
$$

is called a scalar operator.

Definition 2.3. An operator $L$ in $H$ is called a Riesz spectral operator if it can be represented in the form

$$
L=S+N \text {, }
$$

where $S$ is a scalar operator and $N$ is a bounded finite rank nilpotent operator (i.e., there exists an integer $k$ such that $N^{k}=0$ ), which commutes with $S$.

Now we introduce additional assumptions.

If $\operatorname{Im} h=0$, then $\sqrt{p(a) / \rho(a)} \neq|h| ;$ if $\operatorname{Im} k=0, \quad$ then $\sqrt{\rho(0) / p(0)} \neq|k|$.

The main result of the present paper is the following statement.

Theorem 2.1. For any $h, k \in \mathbb{C} \cup\{\infty\}$, both operators $\mathfrak{L}_{h k}$ and $\mathfrak{L}_{h k}^{*}$ are spectral in the sense of Definition 2.3 if conditions (2.3) and (2.11) are satisfied.

REMARK 2.3. a) Conditions (2.3) are not the most general conditions on the coefficients. They can be weakened. b) Conditions (2.11) are essential. As is clear from Theorem 2.1 below, if these conditions fail, then the spectral asymptotics are 
not valid any more. In that case, the Riesz basis property of the root vectors may be lost.

We note that to prove that a particular operator $A$ is Riesz spectral, it suffices to show the following facts:

a) $A$ has a purely discrete spectrum;

b) $A$ may have only a finite number of eigenvectors with finite chains of associated vectors $(\varphi$ is an associated vector of $A$ of the order $m$ corresponding to the eigenvalue $\lambda$ if $\varphi \neq 0$, $(A-\lambda I)^{m} \varphi \neq 0$, and $\left.(A-\lambda I)^{m+1} \varphi=0\right)$

c) the system of the root vectors of $A$ forms a Riesz basis in $H$.

Note that for our operators $\mathfrak{L}_{h k}$, properties a) and b) hold since these operators have compact resolvents. In practice, the proof of fact $\mathrm{c}$ ) is a difficult problem. For the solution of the latter problem, we suggest the method of transformation operators.

Before we define the transformation operators, we would like to mention those particular cases for which the Riesz basis property of the root vectors has been established by other methods.

REMARK 2.4. 1) In our paper [4], it is shown that the set of the root vectors forms a Riesz basis under the following conditions: a) $p=1, \rho \in C^{2}[0, a], \mathfrak{d} \in C^{1}[0, a], k=$ $\infty$ and $h$ is real; and b) the spectrum of $\mathfrak{L}_{h, \infty}$ is simple; i.e., there are no associated vectors. All conditions are important for the proof.

2) In [5-7], it is shown that the set of the root vectors of the operator $\mathfrak{L}_{1, \infty}$ forms a Riesz basis for an undamped string $(\mathfrak{d}=0)$. In [5], the main result is proven for the densities $\rho$ which may have zeros (infinite rarefaction of the matter) or integrable singularities (infinite condensation of the matter: $\rho \rightarrow \infty$ ). The fact that $\mathfrak{d}=0$ is crucial for the proof.

3) In [8], the Riesz basis property of the root vectors has been shown for the case of the Regge problem $(p(x)=1, \mathfrak{d}=0, h=1, k=\infty)$ for the system of ordinary differential equations.

4) In [9], the Riesz basis property of the root vectors has been shown for the operator $\mathfrak{L}_{\infty, \infty}$ under the conditions $\rho=p=1, q=0$ and $\mathfrak{d}$ is a function of bounded variation.

5) In [10], another proof of the Riesz basis property has been suggested in the case of a homogeneous string damped at one end (i.e., for the operator $\mathfrak{L}_{1, \infty}$ under the conditions $p=1, \mathfrak{d}=q=0$ ).

We would like to emphasize that, in spite of the fact that for some particular cases the problem of the Riesz basis property of the set of root vectors has been solved, the combination of a nonconstant density of a string with a nonzero damping makes the problem significantly more complicated. To solve this problem, we use the idea of transformation operators. In fact, this kind of operators was introduced long ago (see B.M. Levitan [23].) Since then, these operators have been successfully used in the study of inverse scattering problem (see [24, 25]). To the best of our knowledge, our paper is the first in which the transformation operators are applied to the proof of the Riesz basis property of the root vectors of a class of nonselfadjoint operators.

3. Statement of Auxiliary Results. In this section, we first reproduce all necessary spectral results from $[4,26]$. Then we introduce the nonselfadjoint quadratic operator pencils associated with the operators $\mathfrak{L}_{h k}$ and $\mathfrak{L}_{h k}^{*}$. We need these pencils in order to recall the definitions of the incoming and outgoing transformation oper- 
ators introduced in [1]. Finally, we formulate those properties of the transformation operators which we need for the proofs of our main results in the present paper.

Our first statement (Theorem 3.1) is a formulation of certain results from our papers $[4,26]$. Actually, the results in the aforementioned papers have been proven for the particular case $k=\infty$. The generalization to an arbitrary complex $k$ can be made without any difficulties by the method developed in those papers.

THEOREM 3.1. a) The operator $\mathfrak{L}_{h k}$ has a countable set of eigenvalues $\left\{\lambda_{n}^{h k}\right\}_{n \in \mathbb{Z}}$. If both parameters $h$ and $k$ satisfy conditions (2.11), then the set of eigenvalues is located in a strip parallel to the real axis and has only two points of accumulation: $+\infty$ and $-\infty$ in the sense that $\operatorname{Re} \lambda_{n}^{h k} \rightarrow \pm \infty$ as $n \rightarrow \pm \infty$ and $\operatorname{Im} \lambda_{n}^{h k} \rightarrow$ const as $n \rightarrow \pm \infty$ (see (3.3) below).

b) For each pair $(h, k)$, satisfying condition (2.11), the following asymptotic formula is valid:

$$
\lambda_{n}^{h k}=\Lambda_{n}^{h k}+O\left(|n|^{-\alpha}\right), \quad|n| \rightarrow \infty,
$$

where $\alpha=1$ if $p, \rho \in C^{2}[0, a]$ and, in general, $0<\alpha<1$ if only conditions (2.3) are satisfied.

In formula (3.1),

$$
\Lambda_{n}^{h k}=\mathcal{M}^{-1}[(n+0.5 \operatorname{sgn} n) \pi+i \mathcal{N}]+\mathcal{D}_{h k},
$$

where

$\mathcal{D}_{h k}=\mathcal{M}^{-1}\left[\left(n+\frac{1}{2} \operatorname{sgn} n\right) \pi+i\left(\mathcal{N}+\frac{1}{2} \ln \frac{\sqrt{p(a) / \rho(a)}+h}{\sqrt{p(a) / \rho(a)}-h}+\frac{1}{2} \ln \frac{\sqrt{\rho(0) / p(0)}-k}{\sqrt{\rho(0) / p(0)}+k}\right)\right]$,

and $\mathcal{M}$ and $\mathcal{N}$ are defined by the formulas:

$$
\mathcal{M}=\int_{0}^{a} \sqrt{\rho(t) / p(t)} d t, \quad \mathcal{N}=\int_{0}^{a} \mathfrak{d}(t) \sqrt{\rho(t) / p(t)} d t
$$

c) All eigenvalues of $\mathfrak{L}_{h k}$ have geometric multiplicities 1 ; i.e., for each $\lambda_{n}^{\text {hk }}$ there exists only one normalized eigenvector $\Psi_{n}$. However, a finite number of eigenvalues $\left\{\lambda_{n}^{h k}, n \in R \in \mathbb{Z}\right\}$ may have finite algebraic multiplicities $\left\{m_{n}\right\}$; i.e, for such $\lambda_{n}^{h k}$ there exists a finite chain of associate vectors $\left\{\Psi_{n}^{j}\right\}_{j=1}^{m_{n}-1}$ :

$$
\left(\mathfrak{L}_{h k}-\lambda_{n}^{h k} I\right) \Psi_{n}^{j}=\Psi_{n}^{j-1}, \quad \Psi_{n}^{0}=\Psi_{n}, \quad \Psi_{n}^{-1}=0 .
$$

Our next step is to recall the definition of the transformation operators introduced in [1]. To do this step, we have to consider the nonselfadjoint quadratic operator pencils associated with the operators $\mathfrak{L}_{h k}$ and $\mathfrak{L}_{h k}^{*}$. The first pencil is defined by the following formula and boundary conditions:

$$
\begin{aligned}
& \mathcal{P}_{h k}(\lambda) \varphi=-L \varphi+\lambda^{2} \varphi-2 i \lambda \mathfrak{d} \varphi \\
& \left(\varphi^{\prime}+i \lambda k \varphi\right)(\lambda, 0)=\left(\varphi^{\prime}+i \lambda h \varphi\right)(\lambda, a)=0
\end{aligned}
$$

where $L$ is given in (2.2). 
To show the relationship between the operator $\mathfrak{L}_{h k}$ and the pencil $\mathcal{P}_{h k}(\lambda)$, we note that the eigenvalue equation for the operator $\mathfrak{L}_{h k}: \mathfrak{L}_{h k} \Psi=\lambda \Psi, \Psi=\left(\begin{array}{c}\psi_{0} \\ \psi_{1}\end{array}\right)$ implies that $\psi_{1}=\lambda \psi_{0}$ and that the function $w=\psi_{0}$ satisfies the eigenvalue equation for the pencil

$$
\mathcal{P}_{h k}(\lambda) w=0, \quad\left(w^{\prime}+i \lambda k w\right)(\lambda, 0)=\left(w^{\prime}+i \lambda h w\right)(\lambda, a)=0 .
$$

We say that $\lambda$ is an eigenvalue of the pencil $\mathcal{P}_{h k}(\lambda)$ if problem (3.8) has a nontrivial solution, which is called then an eigenfunction of $\mathcal{P}_{h k}(\lambda)$. As a consequence, we find that the following fact is valid: the two-component vector $\Psi=\left(\begin{array}{c}\psi_{0} \\ \psi_{1}\end{array}\right)$ is an eigenfunction of $\mathfrak{L}_{h k}$ if and only if the first component $\psi_{0}$ is an eigenfunction of the pencil $\mathcal{P}_{h k}(\lambda)$.

Now we introduce the pencil $\mathcal{P}_{h k}^{*}(\lambda)$ adjoint to $\mathcal{P}_{h k}(\lambda)$ :

$$
\begin{aligned}
& \mathcal{P}_{h k}^{*}(\lambda) \varphi=-L \varphi+\lambda^{2} \varphi+2 i \lambda \mathfrak{d} \varphi, \\
& \left(\varphi^{\prime}-i \lambda \bar{k} \varphi\right)(\lambda, 0)=0, \quad\left(\varphi^{\prime}-i \lambda \bar{h} \varphi\right)(\lambda, a)=0 .
\end{aligned}
$$

To introduce the transformation operators, we have to consider special solutions of the pencil equations: $\mathcal{P}_{h k}(\lambda) w=0$ and $\mathcal{P}_{h k}^{*}(\lambda) w=0$. It is convenient for us to write these equations explicitly:

$$
\left(p(x) w^{\prime}(x)\right)^{\prime}+\lambda^{2} \rho(x) w(x)-2 i \lambda \mathfrak{d}(x) \rho(x) w(x)-q(x) \rho(x) w=0
$$

$$
\left(p(x) w^{\prime}(x)\right)^{\prime}+\lambda^{2} \rho(x) w(x)+2 i \lambda \mathfrak{d}(x) \rho(x) w(x)-q(x) \rho(x) w(x)=0 .
$$

The only difference between these equations is the sign before the terms which are linear in $\lambda$.

Now we have to consider Eqs. (3.11) and (3.12) on the extended interval: $x \in$ $[0, a+\mathcal{M}]$, where $\mathcal{M}$ is given in (3.4). To extend these equations, we assume that

$$
\rho(x)=p(x)=1, \quad \mathfrak{d}(x)=q(x)=0 \text { for } x \in(a, a+\mathcal{M}] .
$$

Note that in [1] in order to introduce the transformation operators, we extended the coefficients to the entire semi-axis $[0, \infty)$. In the present paper, however, only the interval $[0, a+\mathcal{M}]$ is of crucial importance to us. This fact will become clear in Section 4 (see, e.g., Lemma 4.1).

We need also the following functions:

$$
\stackrel{\circ}{E}_{d}^{ \pm}(\lambda, x)=(\rho(x) p(x))^{-1 / 4} \quad e^{ \pm i \nu(\lambda, x)}, \quad \stackrel{\circ}{E}_{-d}^{ \pm}(\lambda, x)=(\rho(x) p(x))^{-1 / 4} \quad e^{ \pm i \nu^{*}(\lambda, x)},
$$

where

$$
\begin{aligned}
& \nu(\lambda, x)=\lambda \int_{0}^{x} \sqrt{\rho(t) / p(t)} d t-i \int_{0}^{x} \mathfrak{d}(t) \sqrt{\rho(t) / p(t)} d t \\
& \nu^{*}(\lambda, x)=\lambda \int_{0}^{x} \sqrt{\rho(t) / p(t)} d t+i \int_{0}^{x} \mathfrak{d}(t) \sqrt{\rho(t) / p(t)} d t .
\end{aligned}
$$


Based on the results of $[4,26]$, we have the following statement.

THEOREM 3.2. Eq. (3.11) has two linearly independent solutions $E_{d}^{ \pm}(\lambda, x)$ with the following approximations:

$$
\left|E_{d}^{ \pm}(\lambda, x)-\stackrel{\circ}{E}_{d}^{ \pm}(\lambda, x)\right| \leq C|\lambda|^{-1}\left|\stackrel{\circ}{E_{d}^{ \pm}}(\lambda, x)\right|
$$

Eq. (3.12) has two linearly independent solutions $E_{-d}^{ \pm}(\lambda, x)$ with the following approximations:

$$
\left|E_{-d}^{ \pm}(\lambda, x)-\stackrel{\circ}{E}_{-d}^{ \pm}(\lambda, x)\right| \leq C|\lambda|^{-1}\left|\stackrel{\circ}{E_{-d}^{ \pm}}(\lambda, x)\right|
$$

For each $x \in[0, a+\mathcal{M}]$, these solutions are holomorphic functions of $\lambda$ for $\operatorname{Im} \lambda>0$ and are continuous for $\operatorname{Im} \lambda \geq 0$.

Now we are in a position to recall the definition of the transformation operators.

Definition 3.1. Linear operators $\mathbb{K}$ and $\hat{\mathbb{K}}$ in the space $H=L_{\rho / p}^{2}(0, a+\mathcal{M})$ which transform the functions $\stackrel{\circ}{E}_{d}^{ \pm}(\lambda, x)$ into the solutions $E_{d}^{ \pm}(\lambda, x)$ of $E q$. (3.11) are called outgoing and incoming transformation operators respectively. [1].

In the next statement - Theorem 3.3 - we represent the main result of our work

THEOREM 3.3. 1) There exist bounded and boundedly invertible operators $\mathbb{K}$ and $\hat{\mathbb{K}}$ in the space $H=L_{\rho / p}(0, a+\mathcal{M})$ such that the following relations are valid:

$$
E_{d}^{+}(\lambda, x)=\left(\mathbb{K}_{E_{d}^{+}}^{+}\right)(\lambda, x), \quad E_{d}^{-}(\lambda, x)=\left(\hat{\mathbb{K}} \stackrel{\circ}{E}_{d}^{-}\right)(\lambda, x)
$$

$\mathbb{K}$ and $\hat{\mathbb{K}}$ are outgoing and incoming transformation operators respectively.

Similar relations are valid for the solutions of the adjoint equation

$$
E_{-d}^{+}(\lambda, x)=\left(\hat{\mathbb{K}} \stackrel{\circ}{E}_{-d}^{+}\right)(\lambda, x), \quad E_{-d}^{-}(\lambda, x)=\left(\mathbb{K}_{E_{-d}^{-}}^{-}\right)(\lambda, x) .
$$

2) The operators $\mathbb{K}$ and $\hat{\mathbb{K}}$ can be represented in the forms

$$
\mathbb{K}=I+\mathfrak{K}, \quad \hat{\mathbb{K}}=I+\hat{\mathfrak{K}},
$$

where $\mathfrak{K}$ and $\hat{\mathfrak{K}}$ are Volterra integral operators given by the following formulas:

$$
(\mathfrak{K} \varphi)(x)=\int_{x}^{\infty} \mathfrak{K}(x, t) \varphi(t) d t, \quad(\hat{\mathfrak{K}} \varphi)(x)=\int_{x}^{\infty} \hat{\mathfrak{K}}(x, t) \varphi(t) d t .
$$

The kernels $\mathfrak{K}$ and $\hat{\mathfrak{K}}$ are continuously differentiable functions with respect to $x$ and $t$. 
4. Riesz Basis Property of the Set of Auxiliary Solutions of the Pencil Equation. In this section, we carry out the first step in the proof of our main result - Theorem 2.1. However, the main result of this section - Theorem 4.2 - might be of interest in itself. To simplify the exposition, we will discuss only the case $k=\infty$ (the homogeneous Dirichlet boundary condition at the left end), but all of the results are valid for any $k \in \mathbb{C} \cup\{\infty\}$.

Now we formulate several definitions.

DeFinition 4.1. 1) The boundary conditions at $x=a$
a) $w^{\prime}(a)+i \lambda h w(a)=0$
b) $w^{\prime}(a)-i \lambda \bar{h} w(a)=0$

are called outgoing and incoming boundary conditions respectively.

2) The solution of Eq. (3.11) satisfying only the outgoing boundary condition is called an outgoing generalized Jost solution and is denoted by $\mathcal{J}_{d}^{>}(\lambda, x)$. The solution of the same equation satisfying the incoming boundary condition is called an incoming generalized Jost solution and is denoted by $\mathcal{J}_{d}^{<}(\lambda, x)$.

3) The solution of Eq. (3.12) satisfying only the outgoing boundary condition is called an adjoint outgoing generalized Jost solution and is denoted by $\mathcal{J}_{-d}^{>}(\lambda, x)$. The solution of the same equation satisfying the incoming boundary condition is called an adjoint incoming generalized Jost solution and is denoted by $\mathcal{J}_{-d}^{<}(\lambda, x)$.

4) Since all of the above generalized Jost solutions are defined up to the multiplication by a constant, we fix them uniquely by imposing the following normalization conditions:

$$
\mathcal{J}_{d}^{>}(\lambda, a)=\mathcal{J}_{d}^{<}(\lambda, a)=\mathcal{J}_{-d}^{<}(\lambda, a)=\mathcal{J}_{-d}^{>}(\lambda, a)=1
$$

It was shown in $[4,26]$ that all these solutions are holomorphic in $\lambda$ for $\operatorname{Im} \lambda>0$ and are continuous for $\operatorname{Im} \lambda \geq 0$ for each $x \in[0, a+\mathcal{M}]$.

REMARK 4.1. We would like to mention the motivation for introducing the aforementioned generalized Jost solutions. In the case $h=1$, the solutions $\mathcal{J}_{\vec{d}}^{>}(\lambda, x)$ and $\mathcal{J}_{-d}^{>}(\lambda, x)$ describe the waves traveling from left to right in the systems governed by Eqs. (3.11) and (3.12) respectively in the so-called Fourier representation. The solutions $\mathcal{J}_{d}^{<}(\lambda, x)$ and $\mathcal{J}_{-d}^{<}(\lambda, x)$ describe the waves traveling from right to left in the systems governed by Eqs. (3.11) and (3.12). The generalization to the cases of arbitrary complex $h$ is important in applications.

Based on the above definitions, we can consider the spectral problems for four quadratic operator pencils. Each of the above pencil spectral problems is defined by the corresponding equation, the outgoing (or incoming) boundary condition at $x=a$ and the Dirichlet boundary condition at $x=0$. We will call the above described pencils by the outgoing (or incoming) pencil and adjoint outgoing (or incoming) pencil. To simplify the exposition, we rewrite the spectral results from Theorem 3.1 for the above described pencils.

THEOREM 4.1. Let

$$
\delta_{ \pm}=\sqrt{p(a) / \rho(a)} \pm h, \quad \sqrt{p(a) / \rho(a)} \neq|h| \quad \text { for real } h .
$$

1) The following formulas are valid for the spectrum $\left\{\lambda_{n}^{>}\right\}_{n \in \mathbb{Z}}$ of the outgoing pencil 
(or the spectrum $\left\{\lambda_{n}^{<}\right\}_{n \in \mathbb{Z}}$ of the incoming pencil):

$$
\begin{aligned}
& \lambda_{n}^{\gtrless}=\Lambda_{n}^{\gtrless}+O\left(|n|^{-\alpha}\right), \quad \text { where } 0<\alpha \leq 1, \text { is the same as in (3.1), } \\
& \Lambda_{n}^{>}=\mathcal{M}^{-1}(n+1 / 2 \operatorname{sqn} n) \pi+i \mathcal{M}^{-1}\left[1 / 2 \ln \left(\delta_{+} \delta_{-}^{-1}\right)+\mathcal{N}\right] \\
& \Lambda_{n}^{<}=\mathcal{M}^{-1}(n+1 / 2 \operatorname{sqn} n) \pi+i \mathcal{M}^{-1}\left[-1 / 2 \ln \left(\overline{\delta_{+} \delta_{-}^{-1}}\right)+\mathcal{N}\right] .
\end{aligned}
$$

2) The following formulas are valid for the spectrum $\left\{{ }^{*} \lambda_{n}^{>}\right\}_{n \in \mathbb{Z}}$ of the adjoint outgoing pencil (or the spectrum $\left\{{ }^{*} \lambda_{n}^{<}\right\}_{n \in \mathbb{Z}}$ of adjoint incoming pencil):

$$
\begin{aligned}
& { }^{*} \lambda_{n}^{\gtrless}={ }^{*} \Lambda_{n}^{\gtrless}+O\left(|n|^{-\alpha}\right), \quad 0<\alpha \leq 1, \\
& { }^{*} \Lambda_{n}^{>}=\mathcal{M}^{-1}(n+1 / 2 \operatorname{sqn} n) \pi+i \mathcal{M}^{-1}\left[1 / 2 \ln \left(\delta_{+} \delta_{-}^{-1}\right)-\mathcal{N}\right], \\
& { }^{*} \Lambda_{n}^{<}=\mathcal{M}^{-1}(n+1 / 2 \operatorname{sqn} n) \pi+i \mathcal{M}^{-1}\left[-1 / 2 \ln \left(\overline{\delta_{+} \delta_{-}^{-1}}\right)-\mathcal{N}\right] .
\end{aligned}
$$

As was shown in our papers [4, 26], the generalized Jost solution (outgoing or incoming) as well as the adjoint generalized Jost solution (outgoing or incoming) can be represented in the form of a linear combination of the solutions $E_{d}^{ \pm}(\lambda, x)$ (or $\left.E_{-d}^{ \pm}(\lambda, x)\right)$. Let us consider, for definiteness, the outgoing generalized Jost solution. For every $h \in \mathbb{C} \cup\{\infty\}$, there exists a pair of coefficients $A_{h}(\lambda)$ and $B_{h}(\lambda)$ such that

$$
\mathcal{J}_{d}^{>}(\lambda, x)=A_{h}(\lambda) E_{d}^{+}(\lambda, x)+B_{h}(\lambda) E_{d}^{-}(\lambda, x) .
$$

We recall $[4,26]$ that the coefficients $A_{h}(\lambda)$ and $B_{h}(\lambda)$ are holomorphic for $\operatorname{Im} \lambda>0$, are continuous for $\operatorname{Im} \lambda \geq 0$, and have the following asymptotic approximations for Im $h \geq 0$ :

$$
\begin{aligned}
& A_{h}(\lambda)=\delta_{-}\left(1+O\left(|\lambda|^{-\alpha}\right)\right)-\delta_{+} e^{2 i \lambda \mathcal{M}+2 \mathcal{N}}\left(1+O\left(\left(|\lambda|^{-\alpha}\right)\right),\right. \\
& B_{h}(\lambda)=\delta_{+} e^{i \lambda \mathcal{M}+\mathcal{N}}\left(1+O\left(|\lambda|^{-\alpha}\right), \quad 0<\alpha \leq 1\right.
\end{aligned}
$$

where $\mathcal{M}, \mathcal{N}$ and $\delta_{ \pm}$are given in (3.4) and (4.3) respectively. The properties of the generalized Jost solutions introduced by Definition 4.1 will be studied in the next section. From formula (4.8), we see that to prove the results about the generalized Jost solutions we have to know the properties of the solutions $E_{d}^{ \pm}(\lambda, x)$.

Let $\left\{\mu_{n}\right\}_{n \in \mathbb{Z}}$ be any sequence from the set of the following four: $\left\{\lambda_{n}^{<}\right\}_{n \in \mathbb{Z}}$ and $\left\{{ }^{*} \lambda_{n}^{<}\right\}_{n \in \mathbb{Z}}$.

LEMMA 4.1. Each set of exponential-like functions $\left\{\stackrel{\circ}{E}_{d}^{ \pm}\left(\mu_{n}, x\right)\right\}_{n \in \mathbb{Z}}$ (see (3.14)) forms a Riesz basis in its closed linear span in $L_{\rho / p}^{2}(0, \mathcal{A})$, where $\mathcal{A}=a+\mathcal{M}$.

Proof. We consider for definiteness the set $\left\{\stackrel{\circ}{E}_{d}^{+}\left(\mu_{n}, x\right)\right\}_{n \in \mathbb{Z}}$. First, we claim that the set of nonharmonic exponentials $\left\{\exp \left(i \mu_{n} x\right)\right\}_{n \in \mathbb{Z}}$ forms a Riesz basis in its closed linear span in $L^{2}(0,2 \mathcal{M})$, where $\mathcal{M}$ is defined in (3.4). This result is an immediate consequence of asymptotic formulas (4.4), (4.6) and of known facts about nonharmonic exponentials (for details see $[4,7,8,27,30]$ ). 
Let $\mathfrak{M}$ be the closed linear span of the set $\left\{\exp \left(i \mu_{n} x\right)\right\}_{n \in \mathbb{Z}}$ in $L^{2}(0,2 \mathcal{M})$ and $\mathfrak{N}$ be the closed linear span of the set $\left\{\stackrel{\circ}{E}_{d}^{+}\left(\mu_{n}, x\right)\right\}_{n \in \mathbb{Z}}$ in $L_{\rho / p}^{2}(0, \mathcal{A})$. We can verify by a direct computation that

$$
\left(\stackrel{\circ}{E}_{d}^{+}\left(\mu_{n},{ }^{\circ}\right), \stackrel{\circ}{E}_{d}^{+}\left(\mu_{m}, \cdot\right)\right)_{L_{\rho / p}^{2}(0, \mathcal{A})}=\left(e^{i \mu_{n} \cdot}, e^{i \mu_{m}}\right)_{L^{2}(0,2 \mathcal{M})} .
$$

Now we use the Bari theorem [28, p. 311] which states that the sequence of vectors $\left\{\psi_{j}\right\}_{j \in \mathbb{Z}}$ forms a Riesz basis in a Hilbert space $H$ if it has the Gram matrix $\left\|\left(\psi_{j}, \psi_{k}\right)\right\|_{k, j=\infty}^{\infty}$ generating bounded and boundedly invertible operator in $H$. As follows from (4.10), the Gram matrices of the systems $\left\{\stackrel{\circ}{E}_{d}^{+}\left(\mu_{n}, x\right)\right\}_{n \in \mathbb{Z}}$ and $\left\{\exp \left(i \mu_{n} x\right)\right\}_{n \in \mathbb{Z}}$ coincide, and, therefore, the set $\left\{\stackrel{\circ}{E}_{d}^{+}\left(\mu_{n}, x\right)\right\}_{n \in \mathbb{Z}}$ is a Riesz basis in $\mathfrak{N}$ if and only if the set of nonharmonic exponentials is a Riesz basis in $\mathfrak{M}$. This concludes the proof of the lemma.

LEMMA 4.2. If the set of exponentials $\left\{\exp \left(i \mu_{n} x\right)\right\}_{n \in \mathbb{Z}}$ is complete in $L^{2}(0,2 \mathcal{M})$, i.e., $\mathfrak{M}=L^{2}(0,2 \mathcal{M})$, then the set $\left\{\stackrel{\circ}{E}_{d}^{+}\left(\mu_{n}, x\right)\right\}_{n \in \mathbb{Z}}$ is complete in $L_{\rho / p}^{2}(0, \mathcal{A})$, and, therefore, it is a Riesz basis in $L_{\rho / p}^{2}(0, \mathcal{A})$.

Proof. Using a contradiction argument, assume that there exists a function $F \in L_{\rho / p}^{2}(0, \mathcal{A})$ such that $F$ is orthogonal to all $\stackrel{\circ}{E}_{d}^{+}\left(\mu_{n},\right), n \in \mathbb{Z}$. So, if $\xi(x)=$ $\int_{0}^{x} \sqrt{\rho(t) / p(t)} d t$, then we have

$$
\int_{0}^{\mathcal{A}} F(x) \rho(x)(p(x))^{-1} \stackrel{\circ}{E}_{d}^{+}\left(\mu_{n}, x\right) d x=\int_{0}^{2 \mathcal{M}} F(x(\xi)) e^{i \mu_{n} \xi} d \xi=0, \quad n \in \mathbb{Z} .
$$

The latter means that there exists the function $f(\xi) \equiv F(x(\xi)) \in L^{2}(0,2 \mathcal{M})$, which is orthogonal to the vectors of the Riesz basis $\left\{e^{i \mu_{n} \xi}\right\}_{n \in \mathbb{Z}}$. This can happen only if $f \equiv 0$. The proof of the lemma is complete.

REMARK 4.2. We would like to mention that the case $\mathfrak{M}=L^{2}(0,2 \mathcal{M})$ has been studied in our paper [4]. It occurs, for example, when $\left\{\mu_{n}\right\}_{n \in \mathbb{Z}}$ is a simple spectrum of the pencil $\mathcal{P}_{h k}(\lambda)$ (no multiple eigenvalues, and, consequently, no associated vectors).

Now we formulate the main result of this section.

THEOREM 4.2. Let $\left\{\mu_{n}\right\}_{n \in \mathbb{Z}}$ be any sequence out of four given in Theorem 4.1. Then each set of solutions $\left\{E_{d}^{ \pm}\left(\mu_{n}, x\right)\right\}_{n \in \mathbb{Z}}$ of Eq. (3.11) forms a Riesz basis in its closed linear span $\mathcal{E}^{+}\left(\right.$or $\left.\mathcal{E}^{-}\right)$in the space $L_{\rho / p}^{2}(0, \mathcal{A})$. If the set of nonharmonic exponentials $\left\{e^{i \mu_{n} x}\right\}_{n \in \mathbb{Z}}$ is complete in $L^{2}(0,2 \mathcal{M})$, (e.g., if the spectrum of the operator $\mathcal{L}_{h k}$ is simple), then $\mathcal{E}^{+}\left(\right.$or $\left.\mathcal{E}^{-}\right)$coincides with $L_{\rho / p}^{2}(0, \mathcal{A})$.

Proof. Let us consider the set $\left\{E_{d}^{+}\left(\mu_{n}, x\right)\right\}_{n \in \mathbb{Z}}$. Our main tool in this proof is Theorem 3.3. By this theorem, there exists the outgoing transformation operator which relates the solution $E_{d}^{+}(\lambda, \cdot)$ of Eq. (3.11) to the exponential-like function $\stackrel{\circ}{E}_{d}^{+}(\lambda, \cdot)$ by the rule:

$$
E_{d}^{+}(\lambda, x)=\left(\mathbb{K}^{\stackrel{\circ}{E}}+(\lambda, x) .\right.
$$

Due to the facts that $\left\{\stackrel{\circ}{E}_{d}^{+}\left(\mu_{n}, x\right)\right\}_{n \in \mathbb{Z}}$ is a Riesz basis in its closed linear span $\mathfrak{N}$ (Lemma 4.1) and that $\mathbb{K}$ is a bounded and boundedly invertible operator, we obtain, 
that the system $\left\{E_{d}^{+}\left(\mu_{n}, x\right)\right\}_{n \in \mathbb{Z}}$ is related to an orthonormal basis through some bounded and boundedly invertible operator. Therefore, this set is a Riesz basis. This completes the proof for the system $\left\{E_{d}^{+}\left(\mu_{n}, x\right)\right\}_{n \in \mathbb{Z}}$. A similar proof works for the system $\left\{E_{d}^{-}\left(\mu_{n}, x\right)\right\}_{n \in \mathbb{Z}}$. To conclude the proof, it remains to refer to Lemma 4.2. Thus, the theorem is shown completely.

Theorem 4.2 admits the following generalization.

Corollary 4.1. If the set of complex points $\left\{\kappa_{n}\right\}_{n \in \mathbb{Z}} i s$ such that the system of exponentials $\left\{e^{i \kappa_{n} x}\right\}_{n \in \mathbb{Z}}$ forms a Riesz basis in $L^{2}(0,2 \mathcal{M})$, then the set of solutions $\left\{E_{d}^{+}\left(\kappa_{n}, x\right)\right\}_{n \in \mathbb{Z}}$ of $E q$. (3.11) forms a Riesz basis in $L_{\rho / p}^{2}(0, \mathcal{A})$.

We would like to emphasize that Corollary 4.1 can be understood as a stability result for a special set of solutions of Eq.(3.11). Namely, with a given sequence of complex points $\left\{\kappa_{n}\right\}_{n \in \mathbb{Z}}$, the set of the corresponding solutions $\left\{E_{d}^{+}\left(\kappa_{n}, x\right)\right\}_{n \in \mathbb{Z}}$ forms a Riesz basis in $L_{\rho / p}^{2}(0, \mathcal{A})$ if and only if the set of nonharmonic exponentials $\left\{e^{i \kappa_{n} x}\right\}_{n \in \mathbb{Z}}$ forms a Riesz basis in $L^{2}(0,2 \mathcal{M})$. Due to the fact that there exists an extensive literature on the geometry of nonharmonic exponentials (see $[6,7,8,27$, $29,30]$ ), we obtain a lot of information on the geometry of the sets of solutions $\left\{E_{d}^{ \pm}\left(\kappa_{n}, x\right)\right\}_{n \in \mathbb{Z}}$. Moreover, if for a particular set $\left\{\kappa_{n}\right\}_{n \in \mathbb{Z}}$, the set of exponentials $\left\{e^{i \kappa_{n} x}\right\}_{n \in \mathbb{Z}}$ is minimal (linearly independent in the case of infinitely many vectors), but not uniformly minimal, let alone the Riesz basis property, then the same is valid for each of the sets $\left\{E_{d}^{ \pm}\left(\kappa_{n}, x\right)\right\}_{n \in \mathbb{Z}}$ in $L_{\rho / p}^{2}(0, \mathcal{A})$. If the set $\left\{e^{i \kappa_{n} x}\right\}_{n \in \mathbb{Z}}$ is only complete in $L^{2}(0,2 \mathcal{M})$, then the same fact holds for $\left\{E_{d}^{ \pm}\left(\kappa_{n}, x\right)\right\}_{n \in \mathbb{Z}}$. Obviously, all of the above takes place for the sets $\left\{E_{-d}^{ \pm}\left(\kappa_{n}, x\right)\right\}_{n \in \mathbb{Z}}$ in the space $L_{\rho / p}^{2}(0, \mathcal{A})$. From all these facts, we immediately obtain another result of importance for the next section.

CoRollary 4.2. Let a sequence $\left\{\mu_{n}\right\}_{n \in \mathbb{Z}}$ be one of the sequences from Theorem 4.1. Let $\mathfrak{N}^{ \pm}$be closed linear spans in $L_{\rho / p}^{2}(0, \mathcal{A})$ of the systems $\left\{E_{d}^{+}\left(\mu_{n}, x\right)\right\}_{n \in \mathbb{Z}}$ and $\left\{E_{d}^{-}\left(\mu_{n}, x\right)\right\}_{n \in \mathbb{Z}}$ respectively. Let ${ }^{\star} \mathfrak{N}^{ \pm}$be closed linear spans in $L_{\rho / p}^{2}(0, \mathcal{A})$ of the systems $\left\{E_{-d}^{+}\left(\mu_{n}, x\right)\right\}_{n \in \mathbb{Z}}$ and $\left\{E_{-d}^{-}\left(\mu_{n}, x\right)\right\}_{n \in \mathbb{Z}}$ respectively. Then there exist bounded and boundedly invertible operators $\mathfrak{P}_{ \pm}$in $L_{\rho / p}^{2}(0, \mathcal{A})$ which map $\mathfrak{N}^{+}$(or ${ }^{\star} \mathfrak{N}^{+}$) onto $\mathfrak{N}^{-}\left(\right.$or $\left.{ }^{\star} \mathfrak{N}^{-}\right)$by the rule:

$$
E_{d}^{+}\left(\kappa_{n}, x\right)=\left(\mathfrak{P}_{+} E_{d}^{-}\right)\left(\kappa_{n}, x\right), \quad E_{-d}^{+}\left(\kappa_{n}, x\right)=\left(\mathfrak{P}_{-} E_{-d}^{-}\right)\left(\kappa_{n}, x\right)
$$

5. Properties of the Generalized Jost Solutions. As follows from Section 4 (Definition 4.1), there exist four generalized Jost solutions: outgoing, incoming, adjoint outgoing and adjoint incoming. Each generalized Jost solution, considered at the corresponding spectral points $\left(\lambda_{n}^{\overrightarrow{<}}\right.$ or $\left.{ }^{*} \lambda_{n} \overrightarrow{<}\right)$, forms the set of eigenfunctions of the outgoing (or incoming) or the generalized outgoing (or generalized incoming) pencil. Now we construct a certain set of functions using the following rule: we take one function $\mathcal{J}(\lambda, x)$ out of the set of the aforementioned four generalized Jost solutions and consider this particular Jost solution at the points $\lambda=\mu_{n}$ where $\left\{\mu_{n}\right\}_{n \in \mathbb{Z}}$ may be any sequence out of four spectral sequences. Furthermore, if the generalized Jost solution matches the chosen spectrum, then $\mathcal{J}\left(\mu_{n}, x\right)$ is simply the eigenfunction of the corresponding pencil. Otherwise, $\left\{\mathcal{J}\left(\mu_{n}, x\right)\right\}_{n \in \mathbb{Z}}$ is just the set of functions in $L_{\rho / p}^{2}(0, \mathcal{A})$. In this section, we study the properties of such sets. In particular, we describe the properties of the sets of eigenfunctions of our pencils. 
THEOREM 5.1. Let $\left\{\mathcal{J}_{n}(x)=\mathcal{J}\left(\mu_{n}, x\right)\right\}_{n \in \mathbb{Z}}$ and $\left\{a_{n}\right\}_{n \in \mathbb{Z}}$ be an arbitrary $\ell^{2}$ sequence of complex numbers. Then there exists a constant $C$ which does not depend on $\left\{a_{n}\right\}_{n \in \mathbb{Z}}$ and such that the following estimate is valid:

$$
\left\|\sum_{n \in \mathbb{Z}} a_{n} \mathcal{J}_{n}\right\|_{L_{\rho / p}^{2}(0, \mathcal{A})} \leq C \sqrt{\sum_{n \in \mathbb{Z}}\left|a_{n}^{2}\right|},
$$

where $\mathcal{A}=a+\mathcal{M}$ and $\mathcal{M}$ is given in (3.4).

Proof. Let us take, for example, as $\mathcal{J}_{n}(x)$ the outgoing generalized Jost solution. We already know (see (4.8)), that for every complex $h$, there exists a pair of coefficients $A_{h}(\lambda)$ and $B_{h}(\lambda)$ such that:

$$
\mathcal{J}_{n}(x) \equiv \mathcal{J}_{d}^{>}(\lambda, x)=A_{h}(\lambda) E_{d}^{+}(\lambda, x)+B_{h}(\lambda) E_{d}^{-}(\lambda, x) .
$$

Using (4.12), we can rewrite (5.2) when $\lambda=\mu_{n}$ in the form:

$$
\mathcal{J}_{n}(x)=A_{h}\left(\mu_{n}\right)\left(\mathfrak{P}_{+} E_{d}^{-}\right)\left(\mu_{n}, x\right)+B_{h}\left(\mu_{n}\right) E_{d}^{-}\left(\mu_{n}, x\right),
$$

where $\mathfrak{P}_{+}$is a bounded linear operator defined on a closed linear span of $\left\{E_{d}^{-}\left(\mu_{n}, x\right)\right\}_{n \in \mathbb{Z}}$ in $L_{\rho / p}^{2}(0, \mathcal{A})$.

Taking into account that $\left\{E_{d}^{-}\left(\mu_{n}, x\right)\right\}_{n \in \mathbb{Z}}$ is a Riesz basis in its closed linear span in $L_{\rho / p}^{2}(0, \mathcal{A})$, we can write:

$$
\begin{aligned}
& \left\|\sum_{n \in \mathbb{Z}} a_{n} \mathcal{J}_{n}\right\| \leq\left\|\sum_{n \in \mathbb{Z}} a_{n} A_{h}\left(\mu_{n}\right) \mathfrak{P}_{+} E_{d}^{-}\left(\mu_{n}, \cdot\right)\right\|+\left\|\sum_{n \in \mathbb{Z}} a_{n} B_{h}\left(\mu_{n}\right) E_{d}^{-}\left(\mu_{n}, \cdot\right)\right\| \\
& \leq C_{1} \sup _{n \in \mathbb{Z}}\left|A_{h}\left(\mu_{n}\right)\right| \sqrt{\sum_{n \in \mathbb{Z}}\left|a_{n}\right|^{2}}+\sup _{n \in \mathbb{Z}}\left|B_{h}\left(\mu_{n}\right)\right| \sqrt{\sum_{n \in \mathbb{Z}}\left|a_{n}\right|^{2}} .
\end{aligned}
$$

All norms in $(5.4)$ are taken in the weighted space $L_{\rho / p}^{2}(0, \mathcal{A})$.

Using explicit formulas (4.9) for the coefficients $A_{h}\left(\mu_{n}\right)$ and $B_{h}\left(\mu_{n}\right)$, we obtain the estimate:

$$
\sup _{n \in \mathbb{Z}}\left\{\left|A_{h}\left(\mu_{n}\right)\right|, \quad\left|B_{h}\left(\mu_{n}\right)\right|\right\} \leq C_{3}<\infty .
$$

Substituting estimate (5.5) into (5.4), we obtain (5.1). Thus, the proof of the theorem is complete.

COROLlaRY 5.1. a) Let us take two different sequences $\left\{\mu_{n}\right\}_{n \in \mathbb{Z}}$ and $\left\{\tilde{\mu_{n}}\right\}_{n \in \mathbb{Z}}$ out of four sequences defined in (4.4) and (4.6). Then there exists a bounded linear operator $S_{1}$ defined on a closed linear span in $L^{2}(0,2 \mathcal{M})$ of the exponential-like functions $\left\{\stackrel{\circ}{E}_{d}^{+}\left(\tilde{\mu}_{n}, x\right)\right\}_{n \in \mathbb{Z}}$, which depends on the above sequences $\left\{\mu_{n}\right\}_{n \in \mathbb{Z}}$ and $\left\{\tilde{\mu}_{n}\right\}_{n \in \mathbb{Z}}$ and acts as follows:

$$
\mathcal{J}_{d}^{>}\left(\mu_{n}, x\right)=\left(S_{1} \stackrel{\circ}{E_{d}^{+}}\right)\left(\tilde{\mu}_{n}, x\right) .
$$

Statement a) admits the following generalization.

b) Let us take two different sequences of complex numbers $\left\{\kappa_{n}\right\}_{n \in \mathbb{Z}}$ and $\left\{\tilde{\kappa}_{n}\right\}_{n \in \mathbb{Z}}$ having the following property: each set of corresponding exponential families $\left\{e^{i \kappa_{n} x}\right\}_{n \in \mathbb{Z}}$ 
and $\left\{e^{i \tilde{\kappa}_{n} x}\right\}_{n \in \mathbb{Z}}$ forms a Riesz basis in its own closed linear span in $L^{2}(0,2 \mathcal{M})$. Then there exists a bounded linear operator $S_{2}$ acting from $L^{2}(0,2 \mathcal{M})$ into $L_{\rho / p}^{2}(0, \mathcal{A})$ by the following rule:

$$
\mathcal{J}_{\vec{d}}^{>}\left(\kappa_{n}, x\right)=\left(S_{2} \stackrel{\circ}{E_{d}^{+}}\right)\left(\tilde{\kappa}_{n}, x\right) .
$$

If both exponential families are complete in $L^{2}(0,2 \mathcal{M})$, then the operator $S_{2}$ has a bounded inverse.

In the next statement, we describe the geometry of the first derivatives of the set $\left\{\mathcal{J}_{n}(x)\right\}_{n \in \mathbb{Z}}$.

THEOREM 5.2. Let $\left\{a_{n}\right\}_{n \in \mathbb{Z}}$ be an arbitrary sequence of complex numbers: $\left\{a_{n}\right\}_{n \in \mathbb{Z}} \in \ell^{2}(\mathbb{Z})$. Then there exists a constant $C^{\prime}$ which does not depend on $\left\{a_{n}\right\}_{n \in \mathbb{Z}}$ and such that the following estimate is valid:

$$
\left\|\sum_{n \in \mathbb{Z}} a_{n} \mu_{n}^{-1} \mathcal{J}_{n}^{\prime}\right\|_{L_{\rho / p}(0, \mathcal{A})}^{2} \leq C^{\prime} \sqrt{\sum_{n \in \mathbb{Z}}\left|a_{n}\right|^{2}} .
$$

Proof. We will prove this theorem for a particular sequence, e.g., for the one related to the outgoing pencil. Thus, we have $\mathcal{J}_{n}(x)=\mathcal{J}_{d}^{>}\left(\mu_{n}, x\right)$. Note, since $\mathcal{J}_{d}^{>}(\lambda, x)$ is a linear combination of the functions $E_{d}^{+}(\lambda, x)$ and $E_{d}^{-}(\lambda, x)$, it suffices to establish the existence of positive constant $C_{1}^{\prime}$ such that

$$
\| \sum_{n \in \mathbb{Z}} a_{n} \mu_{n}^{-1}\left(E_{d}^{ \pm}\left(\mu_{n},\right)^{\prime} \|_{L_{\rho / p}^{2}(0, \mathcal{A})} \leq C_{1}^{\prime} \sqrt{\sum_{n \in \mathbb{Z}}\left|a_{n}\right|^{2}} .\right.
$$

We will prove only one estimate from (5.9) corresponding to $E_{d}^{+}$; the second one can be shown in a similar way. Let us omit for the current proof the superscript "+". To proceed in this proof, we have to recall some of the necessary facts from our paper [1] which will be needed here. As was shown in [1], the function $E_{d}(\lambda, x)$ satisfies the following Volterra integral equation (see Eq. (3.26) from [1])

$$
E_{d}(\lambda, x)=\stackrel{\circ}{E}_{d}(\lambda, x)+\int_{x}^{a} \mathfrak{K}(x, t) \stackrel{\circ}{E}_{d}(\lambda, t) d t, \quad x \leq a .
$$

Since the kernel $\mathfrak{K}(x, t)$ is continuously differentiable (see Theorem 3.3), Eq. (5.10) can be differentiated term-wise, and for the first derivative, the following equation is valid:

$$
E_{d}^{\prime}(\lambda, x)=\stackrel{\circ}{E}_{d}^{\prime}(\lambda, x)-\mathfrak{K}(x, x) \stackrel{\circ}{E}_{d}(\lambda, x)+\int_{x}^{a} \mathfrak{K}_{x}(x, t) \stackrel{\circ}{E}_{d}(\lambda, t) d t,
$$

where for $\stackrel{\circ}{E}_{d}^{\prime}(\lambda, x)$, we have the representation:

$$
\stackrel{\circ}{E}_{d}^{\prime}(\lambda, x)=\left[(i \lambda-\mathfrak{d}(x)) \sqrt{\rho(x) / p(x)}+\left((\rho(x) p(x))^{-1 / 4}\right)^{\prime}(\rho(x) p(x))^{1 / 4}\right] \stackrel{\circ}{E}_{d}(\lambda, x) .
$$


Taking into account the asymptotics of the spectral sequences (Theorem 4.1) from (5.12), we easily obtain the following relation:

$$
\mu_{n}^{-1} \stackrel{\circ}{E}_{d}^{\prime}\left(\mu_{n}, x\right)=\stackrel{\circ}{E}_{d}\left(\mu_{n}, x\right)\left(1+O\left(|n|^{-1}\right)\right) .
$$

Since the functions $\left\{\stackrel{\circ}{E}_{d}\left(\mu_{n}, x\right)\right\}_{n \in \mathbb{Z}}$ are uniformly bounded with respect to $n$ (see $(3.14),(3.15))$, i.e.,

$$
\sup _{n \in \mathbb{Z}} \max _{x \in[0, a]}\left|\stackrel{\circ}{E}_{d}\left(\mu_{n}, x\right)\right|<\infty
$$

we obtain the following sequence of estimates:

$$
\begin{aligned}
& \left|\mathfrak{K}(x, x) \stackrel{\circ}{E}_{d}\left(\mu_{n}, x\right)\right| \leq C_{2}, \quad\left|\int_{x}^{a} \mathfrak{K}_{x}(x, t) \stackrel{\circ}{E}_{d}\left(\mu_{n}, t\right) d t\right| \leq C_{2}, \\
& \left|\mu_{n}^{-1} E_{d}^{\prime}\left(\mu_{n}, x\right)-i \stackrel{\circ}{E}_{d}\left(\mu_{n}, x\right) \sqrt{\rho(x) / p(x)}\right|=O\left(|n|^{-1}\right), \quad n \in \mathbb{Z} .
\end{aligned}
$$

Substituting estimates (5.15) into (5.11), we obtain:

$$
\mu_{n}^{-1} E_{d}^{\prime}\left(\mu_{n}, x\right)=i \stackrel{\circ}{E_{d}}\left(\mu_{n}, x\right)+O\left(|n|^{-1}\right) .
$$

Now we can prove our main estimate (5.9). We have:

$$
\begin{aligned}
& \left\|\sum_{n \in \mathbb{Z}} a_{n} \mu_{n}^{-1} E_{d}^{\prime}\left(\mu_{n}, \cdot\right)\right\|_{L_{\rho / p}^{2}(0, \mathcal{A})} \\
& \leq\left\|\sum_{n \in \mathbb{Z}} a_{n} \stackrel{\circ}{E}_{d}\left(\mu_{n}, \cdot\right)\right\|_{L_{\rho / p}^{2}(0, \mathcal{A})}+\tilde{C}\left\|\sum_{n \in \mathbb{Z}} a_{n}(1+|n|)^{-1}\right\|_{L_{\rho / p}^{2}(0, \mathcal{A})} .
\end{aligned}
$$

Using the facts that $\left\{a_{n}\right\}_{n \in \mathbb{Z}} \in \ell^{2}(\mathbb{Z})$ and the fact that $\left\{\stackrel{\circ}{E}_{d}\left(\mu_{n}, x\right)\right\}_{n \in \mathbb{Z}}$ forms a Riesz basis of its closed span in $L_{\rho / p}^{2}(0, \mathcal{A})$, we obtain $(5.9)$ immediately.

This statement concludes the proof of the theorem.

COROLlARY 5.2. Let $\left\{\mu_{n}\right\}_{n \in \mathbb{Z}}$ be any sequence of complex numbers for which $\left\{\exp \left(i \mu_{n} x\right)\right\}_{n \in \mathbb{Z}}$ is a Riesz basis in $L^{2}(0,2 \mathcal{M})$. Hence, the set $\left\{\stackrel{\circ}{E}_{d}^{+}\left(\mu_{n}, x\right)\right\}_{n \in \mathbb{Z}}$ is a Riesz basis in $L_{\rho / p}^{2}(0, \mathcal{A})$. Let $\left\{W_{n}^{0}(x)\right\}_{n \in \mathbb{Z}}$ be a Riesz basis biorthogonal to the basis $\left\{\stackrel{\circ}{E}_{d}^{+}\left(\mu_{n}, x\right)\right\}_{n \in \mathbb{Z}}$ in $L_{\rho / p}^{2}(0, \mathcal{A})$. Then the following formula is valid for the system $\left\{W_{n}(x)\right\}_{n \in \mathbb{Z}}$ biorthogonal to the set $\left\{E_{d}^{+}\left(\mu_{n}, x\right)\right\}_{n \in \mathbb{Z}}$ :

$$
W_{n}(x)=W_{n}^{0}(x)+\int_{0}^{x} \mathcal{L}(t, x) W_{n}^{0}(t) d t,
$$

where $\mathcal{L}(t, x)$ is the kernel of the integral operator $\mathfrak{L}$ satisfying the equation

$$
(I+\mathfrak{K})^{-1}=I+\mathfrak{L} .
$$

Here $\mathfrak{K}$ is the Volterra integral operator introduced in (3.21). It is clear that Eq. (5.17) can be written in the form:

$$
W_{n}=\left(I+\mathfrak{L}^{*}\right) W_{n}^{0} .
$$


Let us check that $\left\{W_{n}\right\}_{n \in \mathbb{Z}}$ is biorthogonal to $\left\{E_{n} \equiv E_{d}^{+}\left(\mu_{n}, \cdot\right)\right\}_{n \in \mathbb{Z}}$. If $\stackrel{\circ}{E}_{n} \equiv$ $\stackrel{\circ}{E}_{d}^{+}\left(\mu_{n}, \cdot\right)$, then

$$
\begin{array}{r}
\left(E_{n}, W_{m}\right)=\left((I+\mathfrak{K}) \stackrel{\circ}{E}_{n},\left(I+\mathfrak{L}^{*}\right) W_{m}^{0}\right)=\left((I+\mathfrak{L})(I+\mathfrak{K}) \stackrel{\circ}{E}_{n}, W_{m}^{0}\right) \\
=\left(E_{n}^{0}, W_{m}^{0}\right)=\delta_{n m} .
\end{array}
$$

6. Riesz Basis Property of the Root Vectors. Proof of Main Theorem. In this section, we prove the Riesz basis property result for the root vectors of the operator $\mathfrak{L}_{h k}$. We emphasize that we consider a very general case of nonselfadjoint operators: namely, an operator may have a nonzero damping term in the differential expression and any linear nonselfadjoint boundary conditions at both ends. The case with zero internal damping and dissipative boundary conditions was studied in our papers $[5,31]$. In those papers, we have studied the Riesz basis property of the root vectors of the operator

$$
\mathfrak{L}_{h k}^{0}=-i\left(\begin{array}{cc}
0 & 1 \\
L & 0
\end{array}\right),
$$

defined on the domain:

$$
D\left(\mathfrak{L}_{h k}^{0}\right)=\left\{W=\left(w_{0}, w_{1}\right) \in \mathcal{H}: \mathfrak{L}_{h k}^{0} W \in \mathcal{H} ;\left(w_{0}^{\prime}+k w_{1}\right)(0)=0,\left(w_{0}^{\prime}+h w_{1}\right)(a)=0\right\} .
$$

Note that the difference between $\mathfrak{L}_{h k}$ and $\mathfrak{L}_{h k}^{0}$ is the fact that $\mathfrak{d}=0$ in $\mathfrak{L}_{h k}^{0}$. We recall that in [5] we proved our results for three classes of densities $\rho$. For the first class, we studied the problems with smooth positive densities bounded above and below by positive constants. For the second class, we studied the problems involving densities which could go to infinity at some points inside the interval or even at the endpoints (infinite condensation of the matter). For the third class, the densities could go to zero either at several interior points or at the endpoints (infinite rarefaction of the matter). For all three cases of the density, we proved the Riesz basis property of the set of the root vectors of the corresponding dynamics generators. In [5], we have used the so-called functional model for nonselfadjoint operators by Sz. Nagy and C. Foias [32]. However, for the problems involving a combination of singular densities and nonzero internal dampings $(\mathfrak{d} \neq 0)$, the aforementioned functional model does not exist. We hope that using the transformation operators method developed in [1], we can prove that the dynamics generators of such complicated problems are Riesz spectral.

In the present paper, we will use one of the results from [5] to prove the completeness of the root vectors of the operator $\mathfrak{L}_{h k}$ in $\mathcal{H}$, and then, based on the results of Section 5 of this paper, we prove the Riesz basis property result. As is well known, there are two independent problems: the problem of completeness of a certain system of vectors in a Hilbert space and the problem of the Riesz basis property of the same system. Often the first problem is even more difficult than the second one.

In our first statement in this section, we prove the completeness result.

TheOREM 6.1. For any $h, k \in \mathbb{C} \cup\{\infty\}$, the set of the root vectors of the operator $\mathfrak{L}_{h k}$ is complete in $\mathcal{H}$. 
Proof. First of all, we prove the following fact: zero does not belong to the discrete spectrum of $\mathfrak{L}_{h k}$. Using a contradiction argument, we assume that there exists a nontrivial vector $G=\left(\begin{array}{c}g_{0} \\ g_{1}\end{array}\right)$ such that: $\mathfrak{L}_{h k} G=0$. It is easy to verify that the latter equation implies the following:

$$
\begin{aligned}
& \frac{1}{\rho(x)}\left(p(x) g_{0}^{\prime}(x)\right)^{\prime}-q(x) g_{0}(x)-2 \mathfrak{d}(x) g_{1}(x)=0, \\
& \left(g_{0}^{\prime}+k g_{1}\right)(0)=0,\left(g_{0}^{\prime}+k g_{1}\right)(a)=0, g_{1}(x)=0 .
\end{aligned}
$$

Due to the fact that $g_{1}(x)=0$, we have the following boundary-value problem for the component $g_{0}(x)$ :

$$
\left(p(x) g_{0}^{\prime}(x)\right)^{\prime}-q(x) \rho(x) g_{0}(x)=0, \quad g_{0}^{\prime}(0)=g_{0}^{\prime}(a)=0 .
$$

Multiplying Eq. (6.5) by $\overline{g_{0}}$ and integrating over $[0, a]$, we obtain

$$
p(a) g_{0}^{\prime}(a) \bar{g}_{0}(a)-p(0) g_{0}^{\prime}(0) \bar{g}_{0}(0)-\int_{0}^{a} p(x)\left|g_{0}^{\prime}(x)\right|^{2} d x-\int_{0}^{a} q(x) \rho(x)\left|g_{0}(x)\right| d x=0 .
$$

Taking into account the boundary conditions from (6.5), we simplify Eq. (6.6) and obtain

$$
\left\|\sqrt{p} g_{0}^{\prime}\right\|_{L^{2}(0, a)}^{2}+\left\|\sqrt{q \rho} g_{0}\right\|_{L^{2}(0, a)}^{2}=0,
$$

which can happen only if $g_{0}=0$.

Next we notice that

$$
\mathfrak{L}_{h k}=\mathfrak{L}_{h k}^{0}+\mathfrak{D},
$$

where $\mathfrak{L}_{h k}^{0}$ is given in (6.1) and (6.2), and $\mathfrak{D}$ is a bounded linear operator in $\mathcal{H}$ given by the formula

$$
\mathfrak{D}=-i\left(\begin{array}{cc}
0 & 0 \\
0 & -2 \mathfrak{d}
\end{array}\right)
$$

To continue the proof, we have to reproduce some results from $[2,5]$ describing the spectral properties of the operator $\mathfrak{L}_{h k}^{0}$.

THEOREM 6.2. For any $h, k \in \mathbb{C} \cup\{\infty\}$, the following statements hold.

1) $\mathfrak{L}_{h k}^{0}$ has a purely discrete spectrum. Its resolvent is a meromorphic function, with the possibility of having finitely many poles which have finite multiplicities; all others are simple poles.

2) $\left(\mathfrak{L}_{h k}^{0}\right)^{-1}$ exists and is a compact operator of the class $\mathfrak{S}_{p}(\mathcal{H}), p>1$. (We recall that $\mathfrak{S}_{p}(\mathcal{H}), 0<p<\infty$ is the class of all compact operators $A$ in $\mathcal{H}$, which have the following property: the sequence of the eigenvalues of the operator $\left(A A^{*}\right)^{1 / 2}$ belongs to $\ell^{p}$. By $\mathfrak{S}_{\infty}(\mathcal{H})$, we denote the class of all compact operators in $\mathcal{H}$ [28]).

3) The set of all root vectors of $\mathfrak{L}_{h k}^{0}$ forms Riesz basis in $\mathcal{H}$. $\left(\mathfrak{L}_{00}^{0}\right)^{-1}$ :

4) For $h \neq 0$, and $k \neq 0$, the operator $\left(\mathfrak{L}_{h k}^{0}\right)^{-1}$ is a rank-two perturbation of

$$
\left(\mathfrak{L}_{h k}^{0}\right)^{-1}=\left(\mathfrak{L}_{00}^{0}\right)^{-1}+A_{h k},
$$


where $A_{h k}$ is a rank-two operator in $\mathcal{H}$. For $h \neq 0$ and $k=0$ (or $h=0$ and $k \neq 0$ ), the operator $\left(\mathfrak{L}_{h k}^{0}\right)^{-1}$ is a rank-one perturbation of $\left(\mathfrak{L}_{00}^{0}\right)^{-1}$.

Based on Statement 2) of Theorem 6.2, we can show that

$$
\mathfrak{L}_{h k}^{-1}=\left(\mathfrak{L}_{h k}^{0}\right)^{-1}(I+S)
$$

with $S \in \mathfrak{S}_{p}, p>1$. Indeed, due to the existence of the operator $\left(\mathfrak{L}_{h k}^{0}\right)^{-1}$ we can write

$$
\mathfrak{L}_{h k}=(I+V) \mathfrak{L}_{h k}^{0}, \quad V=\mathfrak{D}\left(\mathfrak{L}_{h k}^{0}\right)^{-1},
$$

with $V$ being from $\mathfrak{S}_{p}, p>1$.

Note that $(-1)$ is not an eigenvalue of the operator $V$; otherwise, zero would have been an eigenvalue of the operator $\mathfrak{L}_{h k}$. The fact that zero is not an eigenvalue of $\mathfrak{L}_{h k}$ has been shown at the beginning of the proof of Theorem 6.1. Now we have

$$
(I+V)^{-1}=I+S, \quad S=-V(I+V)^{-1},
$$

and formula (6.10) is justified.

Based on Theorem 6.2 (Statement 4), we obtain that the following equations are valid:

$$
\mathfrak{L}_{h k}^{-1}=\left(\left(\mathfrak{L}_{00}^{0}\right)^{-1}+A_{h k}\right)(I+S)=(I+T)\left(\mathfrak{L}_{00}^{0}\right)^{-1}(I+S),
$$

where $T=A_{h k} \mathfrak{L}_{00}^{0}$ is a rank-two (or rank-one)operator in $\mathcal{H}$, and $S$ is defined in (6.12).

Note that the operator $\mathfrak{L}_{00}^{0}$ corresponds to the problem with zero damping term $(\mathfrak{d}=0)$ and with the Neumann boundary conditions at both ends. So $\mathfrak{L}_{00}^{0}$ is selfadjoint, while $T$ and $S$ are compact but not necessarily selfadjoint.

Now we use the following corollary of the Keldysh Theorem (see [28]).

THEOREM 6.3. Let $A$ be an operator in a Hilbert space $H$ such that $A=(I+$ $\left.S_{1}\right) B\left(I+S_{2}\right)$ where $B=B^{*} \in \mathfrak{S}_{p}, p<\infty$ and $S_{1}, S_{2} \in \mathfrak{S}_{\infty}$. If the operator $A$ vanishes only on the zero vector, then the system of its root vectors is complete in $H$.

If we identify $\mathfrak{L}_{h k}^{-1},\left(\mathfrak{L}_{00}^{0}\right)^{-1}, T$, and $S$ with $A, B, S_{1}$, and $S_{2}$ respectively, then from Theorem 6.3 we obtain the desired completeness in $\mathcal{H}$ of the root vectors of the operator $\mathfrak{L}_{h k}$. Theorem is proven.

Before we prove our main result, we recall that, as was mentioned at the beginning of Section 4 , we have discussed the case $k=\infty$ only to simplify the exposition in that section. All results are valid for any $k \in \mathbb{C} \cup\{\infty\}$. Below we formulate without proofs the results, which are the generalizations for the case of $k \neq \infty$ of the results established in Theorems 5.1 and 5.2 .

THEOREM 6.4. 1) Let $\left\{\lambda_{n}^{h k}\right\}_{n \in \mathbb{Z}}$ and $\left\{\varphi_{n}^{h k}\right\}_{n \in \mathbb{Z}}$ be the spectrum and the set of eigenvectors of the pencil $\mathcal{P}_{h k}(\lambda)$, respectively. Then for any $h, k \in \mathbb{C} \cup\{\infty\}$, there exist bounded linear operators $S_{h k}$ and $T_{h k}$ in the space $L_{\rho / p}^{2}(0, \mathcal{A})$ which act in the following ways:

$$
\begin{aligned}
& \varphi_{n}^{h k}(x)=\left(S_{h k} \stackrel{\circ}{E}_{d}^{+}\right)\left(\lambda_{n}^{h k}, x\right) \\
& \left(i \lambda_{n}^{h k}\right)^{-1}\left(\varphi_{n}^{k k}(x)\right)^{\prime}=\left(T_{h k} \stackrel{\circ}{E}_{d}^{+}\right)\left(\lambda_{n}^{h k}, x\right) .
\end{aligned}
$$


2) Let $\left\{\hat{\varphi}_{n}^{h k}\right\}_{n \in \mathbb{Z}}$ be the set of eigenvectors of the adjoint pencil $\mathcal{P}_{h k}^{*}(\lambda)$. Then there exist bounded linear operators $\hat{S}_{h k}$ and $\hat{T}_{h k}$ in the space $L_{\rho / p}^{2}(0, \mathcal{A})$ which act in the following ways:

$$
\begin{aligned}
& \hat{\varphi}_{n}^{h k}(x)=\left(\hat{S}_{h k} \stackrel{\circ}{E}_{d}^{+}\right)\left(\lambda_{n}^{h k}, x\right), \\
& \left(i \bar{\lambda}_{n}^{h k}\right)^{-1}\left(\hat{\varphi}_{n}^{h k}(x)\right)^{\prime}=\left(\hat{T}_{h k} \stackrel{\circ}{E}_{d}^{+}\right)\left(\lambda_{n}^{h k}, x\right) .
\end{aligned}
$$

3) Let $\left\{\Phi_{n}^{h k}\right\}_{n \in \mathbb{Z}}$ and $\left\{\hat{\Phi}_{n}^{h k}\right\}_{n \in \mathbb{Z}}$ be the sets of eigenvectors of the operators $\mathfrak{L}_{h k}$ and $\mathfrak{L}_{h k}^{*}$ respectively. The following formulas are valid:

$$
\Phi_{n}^{h k}(x)=\left(\begin{array}{cc}
\frac{1}{i \lambda_{n}^{h k}} & \varphi_{n}^{h k}(x) \\
& \varphi_{n}^{h k}(x)
\end{array}\right), \quad \hat{\Phi}_{n}^{h k}(x)=\left(\begin{array}{cc}
\frac{1}{i \bar{\lambda}_{n}^{h k}} & \hat{\varphi}_{n}^{h k}(x) \\
& \hat{\varphi}_{n}^{h k}(x)
\end{array}\right), \quad n \in \mathbb{Z} .
$$

The systems in (6.18) are biorthogonal in $\mathcal{H}$.

Note that the relationship between the associated vectors of the pencil $\mathcal{P}_{h k}(\lambda)$ and the operator $\mathfrak{L}_{h k}$ (as well as for the adjoint pencil and adjoint operator) is more complicated than the biorthogonality relationship (6.18) for the eigenfunctions.

Now we are in a position to prove our main result.

Proof of Theorem 2.1. As was already mentioned, we only need to prove the fact that the set of the root vectors of the operator $\mathfrak{L}_{h k}$ forms a Riesz basis in the energy space. In our proof, we use Theorem 5.3 and the following version of the Bari Theorem (see [28]).

Theorem 6.5. (N.K. Bari) Let the sequence $\left\{F_{n}\right\}_{n \in \mathbb{Z}}$ be complete in a Hilbert space $H$ and $\left\{F_{n}^{*}\right\}_{n \in \mathbb{Z}}$ be the biorthogonal sequence which is also complete in $H$. If for any $F \in H$

$$
\sum_{n \in \mathbb{Z}}\left|\left(F, F_{n}\right)_{H}\right|^{2}<\infty \quad \text { and } \quad \sum_{n \in \mathbb{Z}}\left|\left(F, F_{n}^{*}\right)_{H}\right|^{2}<\infty
$$

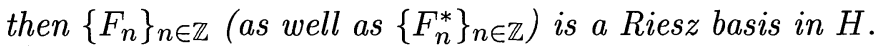

From Theorem 6.1, we have the completeness of the set of the root vectors of the operator $\mathcal{L}_{h k}$ in the space $\mathcal{H}$. Note that the operator $\mathfrak{L}_{h k}^{\star}$ can be obtained from $\mathfrak{L}_{h k}$ if we replace $\mathfrak{d}, h$, and $k$ with $(-\mathfrak{d}),(-\bar{h})$, and $(-\bar{k})$ respectively. This fact allows us to carry out all steps of the proof similar to the ones of Theorem 6.1 with respect to the roots of the operator $\mathfrak{L}_{h k}^{\star}$. Thus, we obtain the completeness result for the operator $\mathfrak{L}_{h k}^{\star}$. Finally, it remains to verify that relations (6.19) hold for both systems: $\left\{\Phi_{n}^{h k}\right\}_{n \in \mathbb{Z}}$ and $\left\{\hat{\Phi}_{n}^{h k}\right\}_{n \in \mathbb{Z}}$. We prove the first estimate from (6.19). The second one can be shown in a similar way. Using (2.7) and (6.18), we have for any $F=\left(\begin{array}{c}f_{0} \\ f_{1}\end{array}\right) \in \mathcal{H}$ :

$$
\begin{aligned}
\left(\Phi_{n}^{h k}, F\right)_{\mathcal{H}}=\left(i \lambda_{n}^{h k}\right)^{-1} \int_{0}^{a} p(x)\left[\left(\varphi_{n}^{h k}(x)\right)^{\prime} \bar{f}_{0}^{\prime}(x)\right. & \left.+q(x) \rho(x) \varphi_{n}^{h k}(x) \bar{f}_{0}(x)\right] d x \\
& +\int_{0}^{a} \rho(x) \varphi_{n}^{h k}(x) \bar{f}_{1}(x) d x
\end{aligned}
$$


Due to the properties of the functions $\rho, p$, and $q$, we arrive at the following estimate:

$$
\left|\left(\Phi_{n}^{h k}, F\right)_{\mathcal{H}}\right|^{2} \leq C\left[\left.\left|\lambda_{n}^{h k}\right|^{-1}\left(\varphi_{n}^{h k}, f_{0}\right)_{H^{1}(0, a)}\right|^{2}+\left|\left(\varphi_{n}^{h k}, f_{1}\right)_{L^{2}(0, a)}\right|^{2}\right],
$$

where $C$ is an absolute constant. According to Theorem 5.2, the following estimate is valid:

$$
\sum_{n \in \mathbb{Z}}\left|\left(\left(\lambda_{n}^{h k}\right)^{-1} \varphi_{n}^{h k}, f_{0}\right)_{H^{1}(0, a)}\right|^{2} \leq \hat{C}_{1}\left\|f_{0}\right\|_{H^{1}(0, a)}^{2}
$$

Theorem 5.1 allows us to conclude

$$
\sum_{n \in \mathbb{Z}}\left|\left(\varphi_{n}^{h k}, f_{1}\right)_{L^{2}(0, a)}\right|^{2} \leq \hat{C}_{2}\left\|f_{1}\right\|_{L^{2}(0, a)}^{2}
$$

Combining (6.22) and (6.23) together, we have shown the first relation of (6.19). Taking into account explicit expression (6.18) for $\hat{\Phi}_{n}^{h k}$ and completing the reasoning analogous to the argument leading to (6.21) - (6.23), we obtain the second inequality of $(6.19)$.

Theorem is completely proven.

Acknowledgment. Partial support by the National Science Foundation Grant DMS-9706882 and Advanced Research Program -97 of Texas Grant \#0036-44-045 is highly appreciated.

\section{REFERENCES}

[1] MARIANnA A. Shubov, Transformation operators for class of domped hyperbolic equations, Preprint TTU, 1999.

[2] MaRianna A. Shubov, Nonselfadjoint operators generated by equation of nonhomogeneous damped string, Transactions of American Mathematical Society, 349:11 (1997), pp. 44814499 .

[3] N. Dunford and J. T. Schwartz, Linear Operators, Part III: Spectral Operators, John Wiley \& Sons, Inc., New York, London, Toronto, 1971.

[4] MARIANNA A. SHUBOv, Basis property of eigenfunctions of nonselfadjoint operator pencils generated by the equation of nonhomogeneous damped string, Int. Eq. and Oper. Theory, 25 (1996), pp. 289-328.

[5] MARIANnA A. Shubov, Asymptotics of resonances and geometry of resonance states in the problem of scattering of acoustical waves by a spherically symmetric inhomogeneity of the density, Dif. Int. Eq., 8:5 (1995), pp. 1073-1115.

[6] M. A. PEKKer (M. A. Shubov), Resonances in the scattering of acoustic waves by a spherical inhomogeneity of the density, Amer. Math. Soc. Transl. (2), 11.5 (1980), pp. 143-163.

[7] S. V. HRUŠČEV, The Regge problem for strings, unconditionally convergent eigenfunctions expansions, and unconditional basis of exponentials in $L^{2}(-T, T)$, J. Oper. Theory, 14 (1985), pp. 67-85.

[8] S. A. Ivanov And B. S. Pavlov, Carleson series of resonances and the Regge problem, Math. USSR Izv., 12:1 (1978), pp. 21-51.

[9] S. COX AND E. ZUAZUA, The rate at which the energy decays in a damped string, Comm. P.D.E., 19:1-2 (1994), pp. 213-243.

[10] S. COX AND E. ZUAZUA, The rate at which the energy decays in a string damped at one end, Indiana Univ. Math. J., 44 (1995), pp. 545-573.

[11] M. S. AGRANOvich, On the convergence of series in the root vectors of almost selfadjoint operators, Trudy Moskov. Math. Obshch., 41 (1980), pp. 163-180; English transl. in Trans. Moscow Math. Soc., 41:1 (1982).

[12] M. S. Agranovich, Spectral properties of diffraction problems, Appendix to N. N. Voitovich, B. Z. Katsenelenbaum, and A. N. Sivov, in The Generalized Method of Eigenoscillations in Diffraction Theory, "Nauka", Moscow, 1997, pp. 289-416. 
[13] Don B. Hinton, An expansion theorem for an eigenvalue problem wirh eigenvalue parameter in the boundary condition, Quatr. J. Math. Oxford Ser. (2), 30 (1979), pp. 33-42.

[14] V. E. KATSNEL'SON, On conditions for basis property of the systems of root vectors for a certain class of operators, Functional. Anal. i Prilozhen., 1:2, pp. 39-51 (1967); English tansl. in Functional Anal. Appl., 1 (1967).

[15] V. B. LIDSKII, On summability of series in the principal vectors of nonselfadjoint operators, Trudy Moskov. Math. Obshch., 11 (1962), pp. 3-35; English transl. in Amer. Math. Soc. Transl. (2), 34 (1964).

[16] A. S. MARCUS, On a basis of root vectors of a disspative operator, Dokl. Akad. Nauk SSSR, 132 (1960), pp. 524-527; English transl. in Soviet. Math. Dokl., 1 (1960).

[17] A. S. MARCUS, On the convergence of multiple expansions in the eigenvectors and associated vectors of an operator pencil, Math. Issled., 4:4(14) (1969), pp. 57-69; English transl. in Amer. Math. Soc. Transl. (2), 110 (1997).

[18] A. S. MARCUS AND V. I. MATSAEV, On the convergence of eigenvector expansions for an operatorwhich is close being selfadjoint, Math. Issled. Vyp., 61 (1981), pp. 104-129. (Russian)

[19] A. S. MARCUS AND V. I. MATSAEV, On the basis property for a certain part of the eigenvectors and associated vectors of an operator pencil, Mat. Sb., 133:175 (1987), pp. 293-313; English transl. in Math. USSR Sb., 61 (1988).

[20] M. V. ORAzov AND G. V. RADzIEVsKII, Theorems on completeness and the basis property for the eigenfunctions of certain regular boundary value problems, Sibirsk. Math. Zh., 17 (1976), pp. 627-639; English transl. in Siberian Math. J., 17 (1976).

[21] A. A. ShKalikov, On the basis property for eigenvectors of quadratic operator pencils, Mat. Zametki, 30 (1980), pp. 371-385; English transl. in Math. Notes, 30 (1980).

[22] A. PAZY, Simigroups of Linear Operators and Applications to Partial Differential Eqautions, Appl. Math. Sciences 44, Springer, New York, 1983.

[23] B. M. Levitan, Theory of Generalized Translation Operators, 2nd Ed., "Nauka", Moscow, 1973.

[24] Z. S. Agranovich and V. A. Marchenko, The Inverse Problem of Scattering Theory, Gordon and Breach, New York, 1967.

[25] V. A. Marchenko, Sturm-Liouville Operators and Applications, Birkhauser, 1986.

[26] Marianna A. Shubov, Asymptotics of resonances and eigenvalues for nohomogeneous damped string, Asymptotic Analysis, 13 (1996), pp. 31-78.

[27] R. M. Young, An Introduction to Nonharmonic Fourier Series, Academic Press, New York, 1980.

[28] I. Ts. Gohberg And M. G. Krein, Introduction to Linear Nonselfadjoint Operators, Trans. of Math. Monogr. 18, AMS, Providence, RI, 1969.

[29] N. K. Nikol'skiI, Treatise on the Shift Operator, Springer-Verlag, Berlin, 1986.

[30] S. V. HRŠČEv, N. K. NiKolSKII, AND B. S. PAVlov, Unconditional bases of exponentials and reproducing kernels, in Lecture Notes in Math. 864, 1981, pp. 214-335.

[31] Marianna A. SHubov, Certain class of unconditional bases in Hilbert space and its applications to functional model and scattering theory, Integral Equations and Operator Theory, 13 (1990), pp. $750-770$.

[32] C. Folas And B. Sz.-NAgy, Harmonic Analysis of Operators in Hilbert Space, Rev. ed., North Holland, Amsterdam, 1970. 
